\title{
Multimodal PTSD Characterization via the StartleMart Game
}

\author{
Christoffer Holmgård - Georgios N. Yannakakis - Héctor P. Martínez • \\ Karen-Inge Karstoft • Henrik Steen Andersen
}

Received: date / Accepted: date

\begin{abstract}
Computer games have recently shown promise as a diagnostic and treatment tool for psychiatric rehabilitation. This paper examines the potential of combining multiple modalities for detecting affective responses of patients interacting with a simulation built on game technology, aimed at the treatment of mental diagnoses such as Post Traumatic Stress Disorder (PTSD). For that purpose, we couple game design and game technology to create a game-based tool for exposure therapy and stress inoculation training that utilizes stress detection for the automatic profiling and potential personalization of PTSD treatments. The PTSD treatment game we designed forces the player to go through vari-
\end{abstract}

Christoffer Holmgård

Center for Computer Games Research

IT University of Copenhagen

Copenhagen, Denmark

E-mail: holmgard@itu.dk

Georgios N. Yannakakis

Institute for Digital Games

University of Malta

Msida, Malta

E-mail: georgios.yannakakis@um.edu.mt

Héctor P. Martínez

Institute for Digital Games

University of Malta

Msida, Malta

E-mail: hector.p.martinez@um.edu.mt

Karen-Inge Karstoft

National Center for Psychotraumatology

University of Southern Denmark

Odense, Denmark

E-mail: kikarstoft@health.sdu.dk

Henrik Steen Andersen

Centre for Crisis and Disaster Psychiatry

Mental Health Services of the Capital Region of Denmark

Copenhagen, Denmark

E-mail: henrik.steen.andersen@regionh.dk ous stressful experiences while a stress detection mechanism profiles the severity and type of PTSD by analyzing the physiological responses to those in-game stress elicitors in two separate modalities: skin conductance (SC) and blood volume pulse (BVP). SC is often used to monitor stress as it is connected to the activation of the sympathetic nervous system (SNS). By including BVP into the model we introduce information about para-sympathetic activation, which offers a more complete view of the psycho-physiological experience of the player; in addition, as BVP is also modulated by SNS, a multimodal model should be more robust to changes in each modality due to particular drugs or day-to-day bodily changes. Overall, the study and analysis of 14 PTSD-diagnosed veteran soldiers presented in this paper reveals correspondence between diagnostic standard measures of PTSD severity and SC and BVP responsiveness and feature combinations thereof. The study also reveals that these features are significantly correlated with subjective evaluations of the stressfulness of experiences, represented as pairwise preferences. More importantly, the results presented here demonstrate that using the modalities of skin conductance and blood volume pulse captures a more nuanced representation of player stress responses than using skin conductance alone. We conclude that the results support the use of the simulation as a relevant treatment tool for stress inoculation training, and suggest the feasibility of using such a tool to profile PTSD patients. The use of multiple modalities appears to be key for an accurate profiling, although further research and analysis are required to identify the most relevant physiological features for capturing user stress.

Keywords stress detection - post traumatic stress disorder · games for health · user profiling 


\section{Introduction}

Post Traumatic Stress Disorder (PTSD) is a psychiatric diagnosis describing an often severely disabling syndrome that is sometimes developed after being exposed to highly stressful situations. Veterans from military operations are a high-risk group for developing this syndrome [1]. A number of psychiatric treatments for PTSD are based on cognitive-behavioral approaches and include exposure therapy and stress inoculation training [2]. Among the possible ways of treating PTSD computer games and virtual environments appear to have a great potential for eliciting stress in a controlled fashion and provide an immersive medium for PTSD treatment facilitating exposure therapy and stress inoculation training. If enhanced with affect detection capabilities, these systems would be able to aid psychiatric evaluation of patients and automatic personalized treatments.

In this paper we investigate the combination of multiple modalities for stress detection in games designed to support the psychiatric treatment of PTSD-diagnosed veteran soldiers. For that purpose, we designed and developed a game - StartleMart — that expands upon existing principles of PTSD treatment techniques with game mechanics and profiles users based on their stress levels, which are inferred from physiological responses to in-game events. In this study, we examine results gathered from 14 veterans diagnosed with PTSD and examine the relation among their PTSD psychiatric profile (measured via standard clinical tools), their perceived stress levels while playing the game (measured via post-experience self-reports), and their physiological responses to in-game stressors (measured via skin conductance (SC) and blood volume pulse (BVP) sensors). Results, building upon and expanding an initial analysis of SC features reported in previous work [3], show that not only SC, but also BVP physiological responses correlate with both PTSD profile features and self-reports of stress. More importantly, results further show that features extracted from the two modalities can be combined into two underlying linear components which are related to measures of PTSD symptom severity. In all, the results demonstrate that capturing user stress responses from multiple physiological modalities enables a more nuanced understanding of patient responses compared to using a single modality. While one could argue that $\mathrm{SC}$ is enough to monitor stress because it is modulated only by the sympathetic nervous system (SNS) — which controls the responses of the body to events perceived as threats - the connection of BVP to both sympathetic and para-sympathetic nervous systems — which in contrast to SNS is linked to relaxation responses - has several advantages. The combination of both signals provides more complete information about the stress responses (e.g. the stress activation and the following relaxation or lack thereof) and more robust monitoring of SNS activations (e.g. motion artifacts, day-to-day changes or effects of drugs more prominent in a single modality [4]).

From the perspective of PTSD treatment, StartleMart represents a novel approach as it uniquely combines real-time stress detection with a game (virtual) environment simulating everyday-life situations. Diverging from and innovating upon earlier work in the use of simulations for treating PTSD [5], we argue that simulating everyday-life situations can help PTSD patients improve their functioning in everyday tasks with direct benefits to their quality of life as a form of stress inoculation training [2]. The present game design expands on previous research and approaches by constructing a desensitization and exposure paradigm consisting of a virtual world representing a home-like setting with integrated game mechanics. The result is a hitherto unexplored midpoint between mediated and in vivo desensitization and exposure paradigms aimed at addressing issues in the everyday-life of the patient. Our experiments show the viability of this approach as both physiological responses and experience self-reports suggest that in-game events can significantly stress soldiers diagnosed with PTSD.

We believe that by interweaving appropriate game design and efficient stress profiling we can provide a personalized therapeutic environment that allows therapists, for the first time, to detect and address common PTSD symptoms across individuals with varying etiologies behind their PTSD. For instance, a veteran soldier and an assault victim may exhibit similar responses to stressful everyday-life situations and a simulation addressing these situations would be relevant to both. Unsurprisingly, the utilization of multiple input modalities appears to be fundamental to empower these solutions with efficient profiling capabilities across individuals.

\section{Stress Detection}

A wide range of approaches exist for capturing stress using physiological, behavioral, and self-report data or combinations thereof. Earlier work on stress detection [6] has demonstrated how features extracted from raw physiological signals can be used to discern between a variety of emotional states in general [7] and in games [8], and previous work has presented designs and studies that build affective loops for PTSD treatment by coupling presented stimuli with PTSD symptom severity $[5,9]$. Informed by this previous research, our con- 
figuration captures indications of stress responses by continuously recording $\mathrm{SC}$ and $\mathrm{BVP}$ and by requesting self-reports from the player.

SC has been identified as a useful indicator of stress elicited from tasks $[10,11]$ and with soldiers [12]. Innervation of the sweat glands is caused solely by the sympathetic nervous system (SNS) whose activation is linked to reaction to threats [13]. By extension, SC activity is related to emotional states such as fear, anger and anxiety, and more generally arousal [14]. Thus, SC is an obvious physiological indicator of player stress.

BVP is a measure of blood flow in body appendices such as finger tips, and it is directly related to heart rate (HR). HR increases with activation of the SNS, but in contrast to SC, HR is also affected by a second control system; HR decreases with activation of the para-sympathetic nervous system (PSNS). This reaction is associated to states of rest and enjoyment [13]. Thus, variability on HR as observed from BVP can reveal changes across both states of stress and relaxation, adding information not easily identified in the SC signal.

Self-reports can provide valuable ground truth [15] for interpreting recorded physiological responses, though they have been shown to be unstable over time and hard to anchor to fixed scales between sessions [16].

For our work presented here, we attempt to exploit $\mathrm{SC}$ to indicate sympathetic activation and HR to indicate para-sympathetic activation with self-report measures as a source of ground truth. In order to mediate the effect of the instability of self-reports, we treat these as expressions of preference rather than direct indications of the subjectively experienced stressfulness.

\subsection{Physiology of PTSD}

In mediated stimulus exposure paradigms, PTSD-patients exhibit physiological responses to stressful visual and auditive stimuli that are significantly different from the responses of non-patients [12]. Their responses are generally characterized by high sympathetic activity as measured by $\mathrm{SC}$ and $\mathrm{HR}$. In experimental studies, slower $\mathrm{SC}$ habituation, elevated resting $\mathrm{SC}$, and greater SC responses to startling stimuli have been found to be robust identifying characteristics of PTSD-patients. Additionally, elevated resting HR and larger HR responses to startling stimuli and trauma cues have been identified as indicators of PTSD. Indeed, HR has been shown to prospectively predict PTSD in some studies [17]. This indicates the higher base levels of arousal and heightened sensitivity to stress that are typical of the disorder. It has been suggested that these differences could be used to support diagnostic differentiation between PTSD patients and non-patients as well as between different degrees of PTSD symptom severity [18] guiding treatment strategies or allowing for adaptive treatment tools [5]. While prior work has related multiple modalities to PTSD, in the present study we contribute by investigating the relationship between PTSD profiles, self-reports of stress and SC, BVP and HR signal features in response to rich interactive simulations and determine that employing and combining multiple physiological modalities provides additional relevant information for characterizing patient responses, compared to using a single modality alone.

\section{The StartleMart Game for PTSD Treatment}

Two well-known treatment approaches for PTSD - favored because of strong evidence for their therapeutic efficacy - are the cognitive-behavioral therapy techniques of exposure therapy and stress inoculation training. In exposure therapy, the therapist confronts the patient with anxiety provoking stimuli in a controlled setting in order to extinguish reactions to the stimuli and/or allow the patient to reprocess the memories cued by the stimuli. Three common variations are the use of real life stimuli i.e. in vivo, representing stimuli via media i.e. mediated, or having the patient imagine the stress provoking situations and thus self-generate the stimuli i.e. imaginal [2]. In stress inoculation training, the therapist exposes the patient to stimuli and situations that are not directly linked to the original trauma of the patient, but that cause problematic anxiety responses that are difficult for the patient to cope with [2]. In the present study we utilize StartleMart as a game facilitator of exposure therapy and stress inoculation training. The game implements a simulation of a number of experiences from everyday life that are known to be stressful to PTSD patients [19], and additionally provides cues of traumatic experiences that war veterans may have experienced. The stimuli are designed around three typical symptoms of PTSD, namely fearavoidance behavior, hyper-arousal (i.e. heightened startle response), and re-experiencing of traumatic events triggered by an outside stimulus or general stress [2]. For a deeper discussion of related work in using simulations and games for mental health, and an in-depth presentation of the StartleMart game, we refer to our previous work [3]. Fig. 1 gives examples of the types of stimuli delivered by the game. 


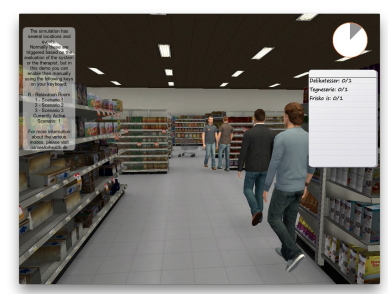

(a) Sound of ventilator blowing overhead.
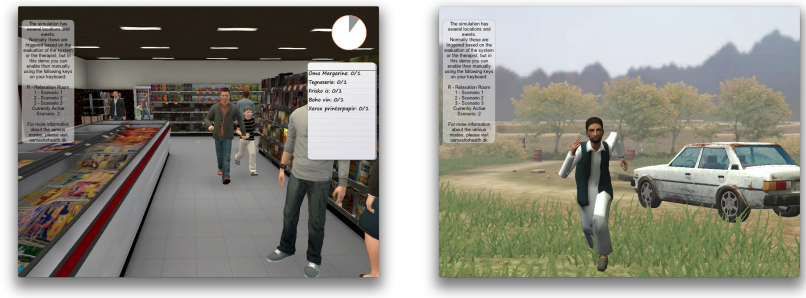

(c) Man walking toward player.(d) Man running toward player.

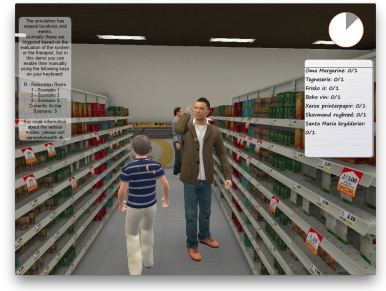

(e) Man staring at player.

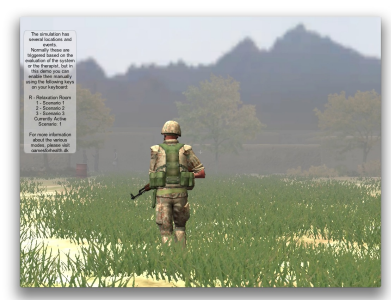

(b) Sound of wind blowing.

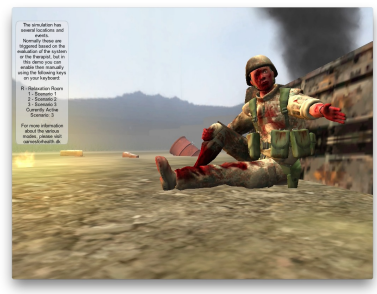

(f) Wounded soldier staring at player.

Fig. 1: The three traumatic experience cues of the game (b, $\mathrm{d}, \mathrm{f})$ and the immediately preceding stressful scenes from everyday life (a, c, e). Elements of the everyday life scenes bleed into the cue scenes, referencing re-experience, a symptom typical for PTSD.

\section{Experimental Protocol and Data Collection}

In this section we provide details about the participants of our experiment and the experimental protocol followed for the clinical trials of the game. Fourteen male PTSD patients, veterans from Danish military operations in Afghanistan, are included in the study presented in this paper. The participants are in psychiatric treatment for PTSD and qualify for the PTSD diagnosis. All subjects in the sample are medicated with $S e$ lective Serotonin Re-uptake Inhibitors (SSRI) which is known to generally lower sympathetic activity and in particular SC [4], while recent research found no significant effect on $\mathrm{HR}$ variability [20]. This clearly adds a challenge to the detection of SC stress responses to game stimuli since patients are expected to manifest responses that are pharmacologically suppressed to an unknown degree. Each patient participates in the experiment twice, engaging in a total of 6 game play ses-

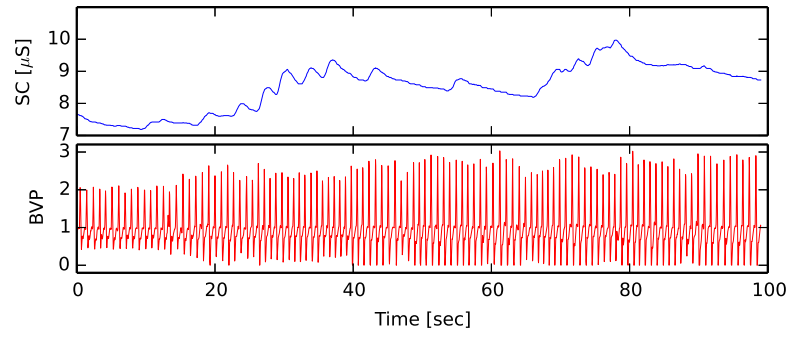

Fig. 2: An example of an SC and a BVP signal recorded from a single game session: Subject no. 5 , game session 3 .

sions, 3 per participation (11 patients have participated in both sessions, while 3 participated in the first session only). For each participation, the 3 sessions vary in terms of goal locations in the virtual environment and in terms of the specific configuration of the stressful experiences.

\subsection{Physiological Sensors and Setup}

For continuous measurement of SC and BVP the IOM biofeedback device ${ }^{1}$ is used. The IOM biofeedback device samples SC and BVP at a rate of $300 \mathrm{~Hz}$ and downsamples the signals to $30 \mathrm{~Hz}$ in firmware before transmitting them to the recording computer. An example of SC and BVP signals collected from a single session is illustrated in Fig. 2. The experimental paradigm and protocol are further detailed in our previous work [3].

\section{User Data Features}

This section details the three types of data obtained from, or extracted for, each experiment participant considered in this study. These include the PTSD profile of the patient, the subjective self-reports of stress during the experiment and the set of features extracted from the physiological signals.

\subsection{PTSD Profile}

Each participant is subjected to the PTSD Module of the Structured Clinical Interview for the DSM (SCID) [21] and completes the military version of the PTSD Checklist-IV (PCL-M) [22], a 17-item questionnaire that yields a PTSD symptom severity score in the interval 17-85. Then all patients are profiled in terms of age, PTSD checklist score $P C L$, number of deployments (i.e. war missions) experienced $N_{d e p}$, and the number

\footnotetext{
1 http://www.wilddivine.com/
} 
of days since their return from their latest deployment $N_{d a y}$. The average, standard deviation and range values of the PTSD profile features across all 14 patients are presented in Table 1 . For the veteran PTSD patients, traumatized by experiences during deployment in this study, we assume that $N_{d a y}$ may be considered an adequately precise measure of the time passed since the traumatizing experience. The deployment situation as a whole may be considered a highly stressful experience and as such part of the traumatizing situation. This means that the age of the trauma for all purposes here is assumed to be equivalent to $N_{d a y}$.

Table 1: PTSD profile features

\begin{tabular}{c|c|c|c}
\hline \hline Feature & Average & Standard deviation & Range \\
\hline Age & 26.8 & 2.5 & $22-32$ \\
PCL & 58.0 & 4.9 & $50-65$ \\
$N_{\text {dep }}$ & 1.77 & 0.67 & $1-3$ \\
$N_{\text {day }}$ & 1001.2 & 432.4 & $113-1685$ \\
\hline \hline
\end{tabular}

\subsection{Self-Reports of Stress}

Before, immediately after, and following a short break after each of the three sessions, the patient is asked to provide a rating of his subjectively experienced stress level on the Subjective Units of Distress Scale (SUDS) [23] in a range from 0 to 100 with 0 representing complete absence of stress and 100 representing the most stressful experience the patient can recall.

\section{Features Extracted from Physiological Signals}

In the following section we present the features extracted from the two physiological signals and the motivation for including these signals. An overview of all features is presented in Table 2 .

\subsection{Features Extracted from Skin Conductance}

SC features are extracted from complete game sessions. Session data is procedurally and visually inspected for outliers and other indications of artifacts. Session data instances containing artifacts are either reconstructed, if possible, or removed from the data set. Following this data cleaning process - that removed $7(9 \%)$ of all possible 75 game sessions resulting in a total of 68 (91\%) sessions - all signals are adjusted for baseline readings, subtracting the individual session mean baseline value from the raw signal. Prior to feature extraction all signals are normalized via min-max normalization within individuals and across sessions from the same day. In order to account for any day-variation effects, signals from the same patients, but taken on different days, are treated as separate individuals. In accordance with recommendations from earlier studies on SC signal processing $[7,8,24]$, a number of features that summarize the key statistical characteristics of SC signals are extracted: Mean SC value $\left(S C_{\bar{x}}\right)$, standard deviation of the SC signal $\left(S C_{\sigma}\right)$, minimum $\mathrm{SC}$ value $\left(S C_{\min }\right)$, maximum $\mathrm{SC}$ value $\left(S C_{\max }\right)$, the difference between the maximum and minimum $\mathrm{SC}$ value $\left(S C_{\text {range }}\right)$, the Pearson correlation between recording time $(t)$ and $\mathrm{SC}$ values $\left(R_{S C t}\right)$, the value of the first $\mathrm{SC}$ sample $\left(S C_{\alpha}\right)$, the value of the final SC sample $\left(S C_{\omega}\right)$, the difference and absolute difference between final and first $\mathrm{SC}$ value $\left(S C_{\omega-\alpha}\right)$ and $\left(\left|S C_{\omega-\alpha}\right|\right)$, the time of the minimum $\mathrm{SC}$ value $\left(t_{S C_{m i n}}\right)$, the time of the maximum SC value $\left(t_{S C_{\max }}\right)$, the absolute time $(t)$ difference between the minimum and maximum SC values $\left(\left|t_{S C_{\text {range }}}\right|\right)$, the means of the absolute values of the first and second differences of the SC signal $\left(S C_{\left|\delta_{1}\right|}\right)$ and $\left(S C_{\left|\delta_{2}\right|}\right)$. An uncommonly used feature, the mean of the absolute first difference of the absolute first difference $\left(\left|S C_{\delta_{\delta}}\right|\right)$, is added in an attempt to describe the tendency toward weak habituation in the signal.

\subsection{Features Extracted from Blood Volume Pulse}

BVP features are also extracted from complete game sessions after inspection for artifacts. Only signals with uncorrupted corresponding SC signals are considered. None of these BVP signals were impacted by artifacts to a detrimental degree and hence BVP features are calculated for all 68 sessions remaining from the first inspection process (91\%). Firstly, heart rate (HR) is computed using a 5 -second sliding window by extrapolating the inter-beat time intervals detected in the BVP signal. The measurement unit for the resulting HR signal is beats per minute (BPM) whereas BVP is a relative measure of blood vessel pressure. Features from $\mathrm{HR}$ as well as BVP are chosen in order to cover the more significant BVP signal dynamics identified in previous studies in the field [25-27]. Note that while HR and $\mathrm{SC}$ present similar features, BVP is a relative signal, and therefore extracted features focus only on its periodic nature. The RR features are aimed at providing an insight on the frequency domain of HR, and they have been developed over decades of research on psychophysiology [26]. 
For HR the following features are extracted: Mean $\left(H R_{\bar{x}}\right)$, maximum $\operatorname{HR}\left(H R_{\max }\right)$, minimum $\operatorname{HR}\left(H R_{\min }\right)$, range of $\mathrm{HR}\left(H R_{\text {range }}\right)$ and standard deviation $\left(H R_{\sigma}\right)$. The Pearson correlation between measurement time and HR value $\left(R_{H R_{t}}\right)$, the $\mathrm{HR}$ at the start of the session $\left(H R_{\alpha}\right)$, at the end of the session $\left(H R_{\omega}\right)$, and the difference between the two $\left(H R_{\omega-\alpha}\right)$. The time of the maximum recorded $\mathrm{HR}$ value $\left(t_{H R_{\text {max }}}\right)$, the time of the minimum recorded $\mathrm{HR}$ value $\left(t_{H R_{\text {min }}}\right)$, and the difference in time between the two $\left(t_{H R_{\text {range }}}\right)$. The local variation of the HR signal as represented by the means of the absolute values of the first and second differences of the signal $\left(H R_{\left|\delta_{1}\right|}\right.$ and $\left.H R_{\left|\delta_{2}\right|}\right)$.

For the raw BVP the following features are extracted: Mean $\left(B V P_{\bar{x}}\right)$, and standard deviation $\left(B V P_{\sigma}\right)$. The local variation of the BVP signal as represented by the means of the absolute values of the first and second differences of the signal $\left(B V P_{\left|\delta_{1}\right|}\right.$ and $\left.B V P_{\left|\delta_{2}\right|}\right)$. The mean and standard deviation of the inter-beat amplitude $\left(I B A m p_{\bar{x}}\right.$ and $\left.I B A m p_{\sigma}\right)$.

Additionally, given the inter-beat time intervals ( $R R$ intervals) of the BVP signal a number of heart rate variability extractors are proposed, concerned with the time-domain and the frequency domain, respectively:

- HRV-time domain: The mean and standard deviation of $\mathrm{RR}$ intervals $\left(R R_{\bar{x}}\right.$ and $\left.R R_{\sigma}\right)$, the fraction of $\mathrm{RR}$ intervals that differ by more than $50 \mathrm{msec}$ from the previous $\mathrm{RR}$ interval ( $p R R 50)$ and the rootmean-square of successive differences of $\mathrm{RR}$ intervals $\left(R R_{R M S}\right)[26]$.

- HRV-frequency domain: The frequency band energy values derived from power spectra obtained using the Lomb periodogram [28]; energy values are computed as the integral of the power of each of the following two frequency bands, relevant for short experiences [29]: High Frequency $(H F)$ band: $(0.15$, $0.4] \mathrm{Hz}$ and Low Frequency $(L F)$ band: $(0.04,0.15]$ Hz. In addition, the ratio $\frac{L F}{H F}$ and the normalized values $\frac{L F}{(L F+H F)}$ and $\frac{H F F}{(L F+H F)}$ are also included as recommended in [29].

\section{Results}

There exists a relation between the PTSD profile of a patient and the levels of stress that is experienced in everyday situations. Therefore we assume a relationship between the patient's PTSD profile and manifestations of stress on the physiological signals recorded across several sessions of interacting with StartleMart. First, we investigate this relation for each modality independently (see Section 7.1 and Section 7.3) using a correlation analysis between the PTSD profile feature set
Table 2: Overview of features extracted from SC and BVP.

\begin{tabular}{|c|c|}
\hline Symbol & Feature \\
\hline$S C_{\bar{x}}$ & Mean SC value \\
\hline$S C_{\max }$ & Max SC value \\
\hline$S C_{m i n}$ & Min SC value \\
\hline$S C_{\text {range }}$ & Difference between max and min SC \\
\hline$S C_{\sigma}$ & Standard deviation of SC \\
\hline$R_{S C t}$ & Correlation, recording time and SC \\
\hline$S C_{\alpha}$ & Value of the first SC sample \\
\hline$S C_{\omega}$ & Value of the final SC sample \\
\hline$S C_{\omega-\alpha}$ & Difference between final and first SC \\
\hline$\left|S C_{\omega-\alpha}\right|$ & Absolute difference between final and first SC \\
\hline$t_{S C_{\max }}$ & Time of the max $\mathrm{SC}$ value \\
\hline$t_{S C_{\min }}$ & Time of the min $\mathrm{SC}$ value \\
\hline$\left|t_{S C_{\text {range }}}\right|$ & Time between the max and min recorded $\mathrm{SC}$ values \\
\hline$S C_{\left|\delta_{1}\right|}$ & Mean of absolute values of $1^{\text {st }}$ difference of SC \\
\hline$S C_{\left|\delta_{2}\right|}$ & Mean of absolute values of $2^{\text {nd }}$ difference of SC \\
\hline$S C_{\left|\delta_{\delta}\right|}$ & $\begin{array}{l}\text { Mean of absolute values of } 1^{\text {st }} \text { difference of } 1^{\text {st }} \text { dif- } \\
\text { ference of SC }\end{array}$ \\
\hline$H R_{\bar{x}}$ & Mean HR \\
\hline$H R_{\max }$ & Max HR \\
\hline$H R_{\min }$ & Min HR \\
\hline$H R_{\text {range }}$ & Range of HR \\
\hline$H R_{\sigma}$ & Standard deviation of $\mathrm{HR}$ \\
\hline$R_{H R_{t}}$ & Correlation, measurement time and $\mathrm{HR}$ value \\
\hline$H R_{\alpha}$ & First HR value of the session \\
\hline$H R_{\omega}$ & Final HR of the session \\
\hline$H R_{\omega-\alpha}$ & Difference between the final and first HR values \\
\hline$t_{H R_{\max }}$ & Time of the max recorded HR value \\
\hline$t_{H R_{\min }}$ & Time of the min recorded HR value \\
\hline$t_{H R_{\text {range }}}$ & Time between max and min recorded HR values \\
\hline$H R_{\left|\delta_{1}\right|}$ & Mean of absolute values of $1^{\text {st }}$ difference of $\mathrm{HR}$ \\
\hline$H R_{\left|\delta_{2}\right|}$ & Mean of absolute values of $2^{\text {nd }}$ difference of $\mathrm{HR}$ \\
\hline$B V P_{\bar{x}}$ & Mean BVP \\
\hline$B V P_{\sigma}$ & Standard deviation of BVP \\
\hline$B V P_{\left|\delta_{1}\right|}$ & Mean of absolute values of $1^{\text {st }}$ difference of BVP \\
\hline$B V P_{\left|\delta_{2}\right|}$ & Mean of absolute values of $2^{\text {nd }}$ difference of BVP \\
\hline$I B A m p_{\bar{x}}$ & Mean of inter-beat amplitude \\
\hline$I B A m p_{\sigma}$ & Standard deviation of inter-beat amplitude \\
\hline$R R_{\bar{x}}$ & Mean of RR intervals \\
\hline$R R_{\sigma}$ & Standard deviation of RR intervals \\
\hline$R R_{R M S}$ & Root-mean-square of differences of RR intervals \\
\hline$p R R 50$ & $\begin{array}{l}\text { Fraction of RR intervals that differ by more than } \\
50 \text { msec from the previous RR interval }\end{array}$ \\
\hline$H F$ & $\begin{array}{l}\text { Integral of power of Lomb periodogram High Fre- } \\
\text { quency band: }(0.15,0.4] \mathrm{Hz}\end{array}$ \\
\hline$L F$ & $\begin{array}{l}\text { Integral of power of Lomb periodogram Low Fre- } \\
\text { quency band: }(0.04,0.15] \mathrm{Hz}\end{array}$ \\
\hline$\frac{L F}{(L F+H F)}$ & Normalized values of the Low Frequency band \\
\hline$\frac{H F}{(L F+H F)}$ & Normalized values of the High Frequency band \\
\hline$\frac{L F}{H F}$ & Ratio of Low Frequency over High Frequency band \\
\hline
\end{tabular}


and the physiological features using Spearman's rank correlation coefficient $\rho$ [30]. Secondly, we use principal component analysis (PCA) to also investigate the interdependencies between modalities by studying the relation between the principal components and the features (see Section 7.5).

Furthermore, we investigate how physiological signals vary along different levels of stress experience during the game. On that basis, we study the correlation between self-reported stress levels and the extracted physiological features using a pair-wise correlation metric (see Section 7.2 and Section 7.4). As noted in Section 2 there is reason to believe that pair-wise preference analysis is a useful approach for examining selfreports. For this purpose, we create two sets of preference pairs. The first set (denoted as Day) contains three preference pairs for each session by comparing the post-experience SUDS ratings given to each of the three games. The preferred game on each pair corresponds to the highest rating (i.e. preference in this context denotes higher stress levels). In cases where the SUDS ratings are equal the stress preference pair is considered ambiguous and discarded. In the second set (denoted as Adjacent), we only extract two pairs from each session following the same procedure. We omit the comparison between the first and third game to minimize noise introduced by the variation on the rating scale due to memory decay. Note that the relations between the self-reported SUDS ratings collected from the patients are expected to become increasingly vague over time. This, in turn, affects the quality of self-reported ratings. Episodic memory traces that form the basis of self-reports fade over time, but the precise rate at which this memory decay occurs is unknown in this case and most likely individual [31]. Ideally, memory decay is so slow that the patient will have a clear feeling of the first session when rating the final session, but it is possible that only comparisons between immediately adjacent sessions are valid. To account for this uncertainty, we analyze the correlations for the Day and Adjacent sets independently. Correlation values are calculated for each physiological feature via the following test statistic [32]

$c(z)=\sum_{i=1}^{N_{s}}\left\{z_{i} / N_{s}\right\}$

where for each pair $i, z_{i}=1$ if the preferred game (i.e. higher stress report) presents a higher feature value, and $z_{i}=-1$ otherwise; $N_{s}$ represents the total number of pairs.

All the correlation coefficients discussed in the following sections are included in Table 3.
Table 3: Correlations $\rho$ between physiological signal features and PTSD profile features are in the left section of the table. Correlations $c(z)$ between physiological signal features and self-reported stress are in the right section of the table. Statistically significant correlations appear in bold $(\mathrm{p}<0.05)$ and italics $(\mathrm{p}<0.10)$.

\begin{tabular}{|c|c|c|c|c|c|c|}
\hline & Age & PCL & $N_{d e p}$ & $N_{d a y}$ & Day & Adjac. \\
\hline$S C_{\bar{x}}$ & 0.10 & 0.10 & 0.01 & 0.08 & 0.15 & 0.14 \\
\hline$S C_{\max }$ & 0.22 & 0.29 & 0.05 & -0.25 & -0.15 & 0.00 \\
\hline$S C_{\min }$ & -0.16 & 0.03 & -0.31 & 0.05 & -0.15 & 0.00 \\
\hline$S C_{\text {range }}$ & 0.23 & 0.24 & 0.13 & -0.26 & -0.25 & -0.19 \\
\hline$S C_{\sigma}$ & 0.26 & 0.17 & 0.13 & -0.23 & -0.15 & 0.00 \\
\hline$R_{S C t}$ & 0.10 & 0.02 & 0.15 & -0.06 & 0.15 & 0.14 \\
\hline$S C_{\alpha}$ & 0.11 & 0.08 & -0.13 & 0.10 & 0.25 & 0.10 \\
\hline$S C_{\omega}$ & 0.08 & 0.35 & -0.17 & -0.30 & -0.02 & -0.05 \\
\hline$S C_{\omega-\alpha}$ & -0.08 & 0.31 & -0.03 & -0.25 & -0.02 & 0.00 \\
\hline$\left|S C_{\omega-\alpha}\right|$ & 0.09 & 0.32 & -0.01 & -0.35 & -0.02 & 0.05 \\
\hline$t_{S C_{\max }}$ & -0.17 & 0.06 & 0.10 & -0.12 & -0.02 & 0.00 \\
\hline$t_{S C_{m i n}}$ & 0.06 & 0.02 & -0.13 & -0.02 & -0.12 & -0.10 \\
\hline$\left|t_{S C_{\text {range }}}\right|$ & -0.04 & -0.07 & 0.11 & -0.15 & 0.08 & 0.05 \\
\hline$S C_{\left|\delta_{1}\right|}$ & 0.15 & 0.29 & 0.13 & -0.26 & -0.12 & -0.14 \\
\hline$S C_{\left|\delta_{2}\right|}$ & 0.15 & 0.28 & 0.14 & -0.25 & -0.12 & -0.14 \\
\hline$S C_{\left|\delta_{\delta}\right|}$ & 0.15 & 0.28 & 0.14 & -0.25 & -0.02 & 0.00 \\
\hline$H R_{\bar{x}}$ & -0.53 & 0.18 & -0.01 & 0.21 & -0.08 & -0.10 \\
\hline$H R_{\max }$ & -0.03 & 0.05 & 0.04 & 0.17 & 0.25 & 0.19 \\
\hline$H R_{m i n}$ & -0.19 & -0.04 & -0.16 & 0.23 & -0.46 & -0.38 \\
\hline$H R_{\text {range }}$ & 0.09 & 0.07 & 0.21 & 0.00 & 0.42 & 0.29 \\
\hline$H R_{\sigma}$ & 0.09 & 0.18 & 0.25 & -0.08 & 0.32 & 0.29 \\
\hline$R_{H R_{t}}$ & 0.19 & 0.02 & 0.05 & -0.33 & -0.05 & -0.05 \\
\hline$H R_{\alpha}$ & -0.07 & -0.05 & 0.07 & 0.31 & -0.05 & 0.05 \\
\hline$H R_{\omega}$ & -0.44 & 0.21 & 0.01 & -0.07 & 0.22 & 0.14 \\
\hline$H R_{\omega-\alpha}$ & -0.21 & 0.15 & -0.07 & -0.25 & 0.12 & 0.00 \\
\hline$t_{H R_{\max }}$ & -0.08 & -0.07 & 0.22 & -0.07 & -0.08 & -0.10 \\
\hline$t_{H R_{\min }}$ & 0.28 & -0.36 & 0.13 & 0.23 & -0.25 & -0.24 \\
\hline$t_{H R_{\text {range }}}$ & -0.26 & 0.22 & 0.07 & -0.15 & 0.19 & 0.24 \\
\hline$H R_{\left|\delta_{1}\right|}$ & -0.08 & 0.39 & 0.17 & -0.25 & 0.32 & 0.38 \\
\hline$H R_{\left|\delta_{2}\right|}$ & -0.11 & 0.40 & 0.19 & -0.24 & 0.32 & 0.33 \\
\hline$B V P_{\bar{x}}$ & -0.09 & 0.28 & 0.02 & -0.16 & 0.05 & 0.00 \\
\hline$B V P_{\sigma}$ & -0.04 & -0.29 & -0.02 & 0.30 & -0.02 & 0.00 \\
\hline$B V P_{\left|\delta_{1}\right|}$ & -0.12 & -0.24 & -0.06 & 0.30 & -0.08 & -0.05 \\
\hline$B V P_{\left|\delta_{2}\right|}$ & -0.12 & -0.24 & -0.06 & 0.30 & -0.08 & -0.05 \\
\hline$I B A m p_{\bar{x}}$ & -0.02 & -0.30 & -0.09 & 0.30 & -0.19 & -0.14 \\
\hline$I B A m p_{\sigma}$ & 0.19 & -0.22 & 0.25 & 0.03 & 0.25 & 0.29 \\
\hline$R R_{\bar{x}}$ & 0.51 & -0.16 & 0.02 & -0.19 & 0.02 & -0.05 \\
\hline$R R_{\sigma}$ & 0.02 & 0.28 & 0.21 & -0.25 & 0.22 & 0.29 \\
\hline$R R_{R M S}$ & -0.03 & 0.31 & 0.19 & -0.33 & 0.19 & 0.24 \\
\hline$p R R 50$ & 0.20 & 0.16 & 0.05 & -0.18 & 0.25 & 0.24 \\
\hline$H F$ & -0.04 & 0.13 & 0.22 & -0.42 & 0.12 & 0.05 \\
\hline$L F$ & 0.17 & -0.23 & -0.25 & 0.31 & -0.46 & -0.38 \\
\hline$\frac{L F}{(L F+H F)}$ & 0.15 & -0.26 & -0.24 & 0.45 & -0.22 & -0.14 \\
\hline$\frac{H F}{(L F+H F)}$ & -0.15 & 0.26 & 0.24 & -0.45 & 0.22 & 0.14 \\
\hline & 0.15 & -0.26 & -0.24 & 0.45 & -0.22 & -0.14 \\
\hline
\end{tabular}


7.1 Correlations Between PTSD Profile and SC Features

Results suggest that patients suffering from more severe degrees of PTSD (higher PCL values) respond with higher $S C_{\max }$ and a higher increase across the sessions as indicated by $S C_{\omega-\alpha}$. This corresponds to findings that PTSD patients are more responsive to stressful stimuli. They also complete the session with a higher $S C_{\omega}$ which corresponds to findings that PTSD patients are more responsive and habituate slower than nonpatients. Patients with more severe PTSD exhibit higher values of all typical measures of local variation. The correlations between PCL and $S C_{\left|\delta_{1}\right|}, S C_{\left|\delta_{2}\right|}$ indicate that patients with more severe PTSD exhibit more variation. We hypothesize this is due to the relation between the severity of the syndrome and the hyper-responsiveness and hyper-arousal of the patient, meaning the patient responds more often to stimuli in the game. $S C_{\left|\delta_{\delta}\right|}$ also correlates with symptom severity suggesting PTSD patients' slower habituation compared to non-patients [17]. Significant positive correlation is observed between $N_{d e p}$ and $S C_{m i n}$. No clear explanation can be given for this, since more deployments should mean a higher degree of exposure to potentially highly stressful situations, but it should be noted that the range of the number of deployments in the sample is limited to 1 to 3 . One could speculate that individuals who were only diagnosed with PTSD after several deployments were less susceptible to contracting the hyper-aroused state of PTSD. It would follow that they would exhibit lower SC bounds than their more susceptible colleagues, but the explanation remains speculation. A negative correlation is observed between $N_{d a y}$ and the last SC value recorded in session; PTSD symptoms typically abate as a function of time [2], so this relation matches the literature on PTSD. The literature also matches the relation between $N_{d a y}$ and PCL: PCL and $N_{d a y}$ correlate negatively $(\rho=-0.51, p<0.01)$ indicating the symptom severity decreases over time. It seems plausible that $N_{d a y}$ is an inverse indicator of symptom severity and that less severe cases of PTSD exhibit lower bounds of SC, most likely due to a less elevated mean SC level and faster (closer to normal) habituation. Altogether, we argue the results indicate a positive relationship between symptom severity and features of SC responses to StartleMart.

\subsection{Correlations Between Self-Reports and SC}

Features

Two significant effects are identified across the two approaches to generating preferences pairs: A negative correlation between self-reports of stress and the range of the SC signal ( $S C_{\text {range }}$ ) and a positive correlation between reported stress and initial $\mathrm{SC}$ values. Both effects are consistent with the fact that patients with severe PTSD symptoms exhibit high SC values and weaker habituation. This means their SC values stay higher and their signals are subject to quick stabilization at the individually higher baseline. The correlations indicate that patients feeling stressed by interacting with StartleMart exhibit matching SC responses and supports the relevance of the game to the target group.

\subsection{Correlations between PTSD Profile and HR/BVP Features}

A number of correlations are observed between the patients' PTSD profiles, and the BVP/HR features. Both average $\left(H R_{\bar{x}}\right)$ and last $\mathrm{HR}\left(H R_{\omega}\right)$ are negatively correlated with age while no significant correlation is observed with respect to PCL, days from last deployment $\left(N_{d a y}\right)$ or days deployed $\left(N_{d e p}\right)$. Age and PCL present an equivalent negative correlation $(\rho=-0.52, p<0.01)$. This could indicate that in this sample older patients exhibit greater resilience toward PTSD as seen by lower PCL scores and lower HR values; an interpretation which is consistent with findings in the literature on PTSD in veterans [33].

More severe PTSD appears to result in a higher reactivity to the stressors as suggested by the positive correlation between PCL and a number of features that measure the local variability of the HR signal $\left(H R_{\left|\delta_{1}\right|}\right.$, $H R_{\left|\delta_{2}\right|}, R R_{\sigma}$ and $\left.R R_{R M S}\right)$. Note that a higher value of these features is typically related to a larger number of peaks in the signal (quick increments on $\mathrm{HR}$ ) that increase local variability while not necessarily affecting global variability (as measured by $H R_{\sigma}$ ). This appears to be a strong relation as it has also been observed in the SC features. Due to the periodicity of BVP, its standard deviation $\left(B V P_{\sigma}\right)$ captures information of different nature, related more closely to the average interbeat amplitude $\left(I B A m p_{\bar{x}}\right)$ than to the local variability of HR. PCL is negatively correlated to both $B V P_{\sigma}$ and $I B A m p_{\bar{x}}$ which suggests that more severe PTSD would be related to higher sympathetic arousal.

PCL is also correlated (negatively) to the time to the lowest recorded HR $\left(t_{H R_{m i n}}\right)$ suggesting that patients with more severe symptoms of PTSD respond earlier to the stressful stimuli and do not revert to a less stressed state during a session, though this feature, as noted, correlates with age as well.

The number of days from deployment $\left(N_{\text {days }}\right)$ appears to be positively correlated with a higher activity of the sympathetic nervous system (captured by $L F$ 
and $\frac{L F}{L F+H F}$ [34], also $I B A m p_{\bar{x}}$ and $\left.B V P_{\sigma}\right)$, negatively correlated to higher activity of the para-sympathetic nervous system (captured by $H F$ and

$\left.\frac{H F}{L F+H F}\right)$ and positively correlated with a dominance of sympathetic over para-sympathetic $(L F / H F)$. Given the connection between sympathetic activity and stress, these results show that the participants with older traumas appear to be more stressed during the therapy than patients with more recent traumas. These correlations to a certain extent run counter to the idea of spontaneous PTSD recovery over time, though one possible explanation could be that patients who are further into treatment respond with stronger manifestations of sympathetic dominance when subjected to novel therapeutic methods. On the other hand, a correlation is observed between $R R_{R M S}$ and $N_{d a y}$. It would seem that this correlation matches the assumption that $N_{d a y}$, representing the age of the trauma, is a rough measure of spontaneous recovery leading to lower manifestation from patients with older traumas. Finally, mean interbeat intervals $\left(R R_{\bar{x}}\right)$ correlate positively with age, mirroring the relation found between $H R_{\bar{x}}$ and age.

\subsection{Correlations between Self-Reports and HR/BVP Features}

Similar patterns of significant effects are identified across the two approaches to generating preferences pairs for the HR/BVP signals. For HR features, measures connected with stress and sensitivity to stress exhibit positive correlations to the ranked subjective evaluation of session stressfulness. The same patterns are observed for the features extracted directly from the BVP signal. Again, as was the case for the SC signal, these correlations indicate both that patients feel stressed from interacting with StartleMart and that this experience scales with symptom severity. The stronger effect between the features derived from HR and self-reports than between the SC features and self-reports matches findings in the literature suggesting that HR features provide a robust physiological indicator of PTSD symptom severity [17].

\subsection{Principal Component Analysis of Physiological} Features and their Relations to PTSD symptom severity

While the results suggest that the applied modalities are useful in characterizing player stress responses in relation to interacting with StartleMart, the high number of features makes the identification of the underlying causes difficult. In order to investigate whether any

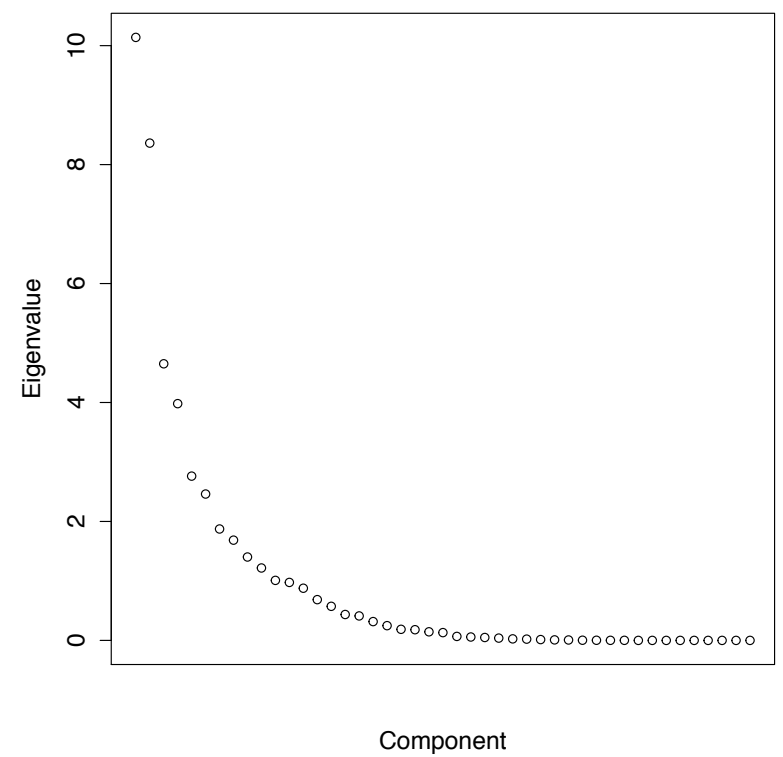

Fig. 3: Scree plot of all 45 principal components derived from the original feature set. The scree plot shows two components explaining most of the variance in the data with the remaining components explain relatively less.

unifying components exist which underlie the correlations in the large feature set, a principal component analysis with no rotation is conducted. All extracted features are subjected to the analysis producing components that combine information across modalities, producing an initial set of 45 principal components.

Initially, 45 principal components are generated. The first five components account for approximately $43 \%$, $30 \%, 12 \%, 7 \%$, and $4 \%$ of the variance, respectively, after which the proportion of explained variance for each component reduces rapidly. The components are depicted in a scree plot in Fig. 3. In order to retain a low number of components, we coerce the model to produce two components, though three components could have been considered as well. However, choosing two components yields a balance between the variance explained by each of the two resulting components as each accounts for approximately half of the variance as indicated in Table $4\left(d f=901, \chi^{2}=16166.24, p<0.01\right)$.

Table 4: Standard deviation and explained variance for each of the two principal components extracted from the feature set.

\begin{tabular}{rrr}
\hline \hline & Component 1 & Component 2 \\
\hline Standard deviation & 3.1841 & 2.8916 \\
Proportion of Variance & 0.5480 & 0.4520 \\
Cumulative Proportion & 0.5480 & 1.0000 \\
\hline \hline
\end{tabular}


Though the analysis does not provide any inherent labeling of the resulting components it could be hypothesized that the two components are related to parasympathetic and sympathetic activation in response to the simulation, respectively, or put differently, the player's ability to habituate to the stimuli or respond to the stimuli with manifestations of stress.

To provide an insight into the most important features for each component, Table 5 presents the Pearson correlations between the individual features and the principal components, ordered by the magnitude of the correlations. Component 1 is characterized primarily by negative correlations to measures of sympathetic activity captured via BVP expressed in HR. As described in Section 2.1, PTSD symptom severity is characterized by elevated resting $\mathrm{HR}$ and larger $\mathrm{HR}$ responses to stimuli and the component appears to capture this phenomenon. Component 2 is characterized primarily by positive correlations to features extracted from SC, indicating sympathetic activity, also in accordance with expectations from the literature. From the correlations between the individual features and the two components, it seems that the first component primarily represents responses captured via BVP, while the second component primarily represents responses captured via SC. However, the fact that both components do exhibit correlations to features from both modalities suggests that the two modalities together enable the capturing of both resilience and sensitivity to manifesting stress in response to the simulation. The principal components are subsequently correlated to Age, PCL, $N_{d a y}$, and $N_{d e p}$, and self-reports as the external measures of symptom severity and tendency to manifest stress in response to the simulation.

To test the relation between these two principal components and the measures of PTSD symptom severity, the same correlation analyses applied to the individual features are applied to the components. The results are reported in Table 6 . A negative correlation between the first component and PCL and a borderline significant positive correlation between the second component and PCL are observed. Additionally, a positive correlation between the first component $N_{d a y}$ is observed, while a negative correlation between the second component and $N_{d a y}$ is evident. The two pairs of correlations conform to expectations from the literature on PTSD symptoms, as described earlier, and could be seen as further indication that the two components represent patients' tendencies toward para-sympathetic and sympathetic activation in response to the simulation. Notably, neither of the components correlate with Age or $N_{\text {dep }}$. Pairwise correlations between the extracted components and self-reports of experienced stress also ex-
Table 5: Correlations between individual features and the two extracted principal components. For each component, the features listed are sorted according to the absolute value of the correlation coefficient in order to allow for a straightforward identification of the features with the strongest relation to the component.

\begin{tabular}{|c|c|c|c|}
\hline \multicolumn{2}{|c|}{ Component 1} & \multicolumn{2}{|c|}{ Component 2} \\
\hline Feature & $R$ & Feature & $R$ \\
\hline$R R_{\sigma}$ & -0.83 & $S C_{\sigma}$ & 0.78 \\
\hline$R R_{R M S}$ & -0.83 & $S C_{\text {range }}$ & 0.78 \\
\hline$H R_{\left|\delta_{1}\right|}$ & -0.77 & $\left|S C_{\omega-\alpha}\right|$ & 0.78 \\
\hline$H R_{\left|\delta_{2}\right|}$ & -0.76 & $S C_{\left|\delta_{1}\right|}$ & 0.77 \\
\hline$L F$ & 0.74 & $S C_{\left|\delta_{2}\right|}$ & 0.77 \\
\hline$\frac{H F}{(L F+H F)}$ & -0.69 & $S C_{\left|\delta_{\delta}\right|}$ & 0.77 \\
\hline$\frac{L F}{(L F+H F)}$ & 0.69 & $S C_{\max }$ & 0.73 \\
\hline$H R_{\text {range }}$ & -0.66 & $S C_{m i n}$ & -0.66 \\
\hline$\frac{L F}{H F}$ & 0.66 & $H R_{\min }$ & -0.62 \\
\hline$H R_{\sigma}$ & -0.63 & $R R_{\bar{x}}$ & 0.52 \\
\hline$I B A m p_{\bar{x}}$ & 0.60 & $H R_{\bar{x}}$ & -0.50 \\
\hline$p R R 50$ & -0.53 & $S C_{\omega-\alpha}$ & 0.48 \\
\hline$S C_{\min }$ & -0.53 & $p R R 50$ & 0.46 \\
\hline$S C_{\text {range }}$ & 0.52 & $S C_{\omega}$ & 0.43 \\
\hline$H R_{m i n}$ & 0.50 & $R R_{\sigma}$ & 0.42 \\
\hline$S C_{\bar{x}}$ & -0.49 & $\frac{L F}{(L F+H F)}$ & -0.42 \\
\hline$B V P_{\left|\delta_{1}\right|}$ & 0.49 & $\frac{H F}{(L F+H F)}$ & 0.42 \\
\hline$B V P_{\left|\delta_{2}\right|}$ & 0.49 & $R R_{R M S}$ & 0.42 \\
\hline$\left|S C_{\omega-\alpha}\right|$ & 0.48 & $H F$ & 0.39 \\
\hline$H R_{\max }$ & -0.48 & $H R_{\omega}$ & -0.38 \\
\hline$S C_{\sigma}$ & 0.43 & $H R_{\alpha}$ & -0.37 \\
\hline$B V P_{\sigma}$ & 0.41 & $\frac{L F}{H F}$ & -0.37 \\
\hline$S C_{\max }$ & 0.41 & ${ }_{S C}^{H} C_{\alpha}$ & -0.35 \\
\hline$S C_{\omega-\alpha}$ & 0.39 & $B V P_{\left|\delta_{2}\right|}$ & -0.33 \\
\hline$S C_{\alpha}$ & -0.39 & $B V P_{\left|\delta_{1}\right|}$ & -0.33 \\
\hline$\left|t_{S C_{\text {range }}}\right|$ & -0.35 & $S C_{\bar{x}}$ & -0.32 \\
\hline$t_{H R_{\max }}$ & 0.35 & $L F$ & -0.32 \\
\hline$t_{H R_{\text {range }}}$ & 0.33 & $I B A m p_{\bar{x}}$ & -0.28 \\
\hline$R_{S C t}$ & -0.33 & $I B A m p_{\sigma}$ & -0.26 \\
\hline$S C_{\omega}$ & 0.32 & $H R_{\text {range }}$ & 0.25 \\
\hline$S C_{\left|\delta_{1}\right|}$ & 0.32 & $H R_{\left|\delta_{1}\right|}$ & 0.23 \\
\hline$S C_{\left|\delta_{2}\right|}$ & 0.32 & $H R_{\left|\delta_{2}\right|}$ & 0.22 \\
\hline$S C_{\left|\delta_{\delta}\right|}$ & 0.32 & $t_{S C_{\max }}$ & 0.19 \\
\hline$H F$ & -0.31 & $t_{S C_{\min }}$ & 0.16 \\
\hline$B V P_{\bar{x}}$ & -0.30 & $R_{S C t}$ & 0.14 \\
\hline$H R_{\bar{x}}$ & -0.29 & $H R_{\sigma}$ & 0.13 \\
\hline$R R_{\bar{x}}$ & 0.25 & $t_{H R_{\max }}$ & 0.12 \\
\hline$I B A m p_{\sigma}$ & -0.24 & $I B A m p_{\sigma}$ & 0.11 \\
\hline$H R_{\alpha}$ & -0.23 & $t_{H R_{\text {range }}}$ & 0.10 \\
\hline$t_{S C_{\min }}$ & 0.15 & $H R_{\max }$ & -0.10 \\
\hline$R_{H R_{t}}$ & 0.14 & $\left|t_{S C_{\text {range }}}\right|$ & 0.09 \\
\hline$H R_{\omega-\alpha}$ & 0.14 & $B V P_{\bar{x}}$ & 0.06 \\
\hline$t_{H R_{\min }}$ & -0.11 & $H R_{\omega-\alpha}$ & 0.06 \\
\hline$H R_{\omega}$ & -0.08 & $R_{S C t}$ & -0.05 \\
\hline$t_{S C_{\max }}$ & 0.02 & $t_{H R_{\min }}$ & -0.02 \\
\hline
\end{tabular}

hibit to the same pattern, with component 1 correlating negatively with reports of stress and component 2 correlating positively with reports of stressful experience. Taken together, this could indicate a more robust relation between the multimodal components, symptom severity, and self-reports than between individual features and external measures of ground truth. 
Table 6: Correlations $\rho$ and $c(z)$ between the two principal components and Age, PCL, $N_{d a y}$, and $N_{d e p}$, and self-reports. Statistically significant correlations appear in bold $(\mathrm{p}<0.05)$ and italics $(\mathrm{p}<0.10)$.

\begin{tabular}{rrr}
\hline \hline & Component 1 & Component 2 \\
\hline Age & 0.10 & 0.18 \\
$\mathrm{p}$ & 0.47 & 0.39 \\
\hline$P C L$ & $\mathbf{- 0 . 2 9}$ & 0.26 \\
$\mathrm{p}$ & 0.04 & 0.06 \\
\hline$N_{d a y}$ & $\mathbf{0 . 3 0}$ & $\mathbf{- 0 . 3 5}$ \\
$\mathrm{p}$ & 0.03 & 0.02 \\
\hline$N_{d e p}$ & -0.22 & 0.21 \\
$\mathrm{p}$ & 0.22 & 0.22 \\
\hline Self-reports, day & $\mathbf{- 0 . 2 5}$ & $\mathbf{0 . 2 2}$ \\
$\mathrm{p}$ & 0.02 & 0.03 \\
\hline Self-reports, adjac. & -0.14 & 0.19 \\
$\mathrm{p}$ & 0.08 & 0.06 \\
\hline \hline
\end{tabular}

\section{Discussion}

The PCL score of the patients served as the first measure of ground truth describing symptom severity in this study. The PCL instrument is well-validated and the de facto standard for PTSD severity screening [2], but is nonetheless based on self-reports of personal experience retrieved from memory. This is an inherent weakness of the presented study, but one we suspect is innate and difficult to overcome in any study involving a syndrome defined partially by personal experience. The negative correlation between PCL values and $N_{\text {day }}$, which matches expectations according to the literature, strengthens the validity of the measure. The second measure of ground truth is the SUDS values collected during the game-play sessions. These are subject to the concerns related to ratings (as described in Sections 2 and 7), but these concerns are sought mediated by the use of pair-wise preferences as the basis for the correlation analysis; this analysis ignores the exact value of the ratings and considers only the ordinal relation between ratings given on the same day or adjacent sessions. In Table 3 negative correlations are present between self-reports and $S C_{\max }$ and $S C_{\min }$ when pairs are constructed across all sessions in a day. Based on findings in the literature, we would expect these to be positive. However, when pairs are limited to adjacent sessions these effects disappear and only effects matching expectations from theory remain. We consider this a confirmation that the absolute value of self-reported ratings of stress becomes increasingly unreliable over time as memories decay. Future work using StartleMart might benefit from including stress evaluations as preferences at the report level.

Some features extracted from BVP indicate dominance of sympathetic activation over para-sympathetic that scales with the age of the trauma, contrary to our expectation of spontaneous recovery. Though one expla- nation could be that veteran patients respond stronger to novel treatment methods, further investigation is necessary to fully understand these relations.

The feature combination through principal component analysis suggests that it may be feasible to reduce the physiological stress manifestations in response to the simulation to two underlying components which could be interpreted as resilience and sensitivity towards the stressful stimuli. These two components correlate with measures of symptom severity and self-reports as expected from the literature. However, as a crossmodality feature combination technique the principal component analysis seems to fall short, as one component is dominated by $\mathrm{BVP} / \mathrm{HR}$ features, and the other component is dominated by SC features.

In general, the analyses presented in this paper are limited to correlating features and applying linear methods of feature combination through principal component analysis. Recent work in the literature $[11,35]$ describes how applications of non-linear techniques of analysis and machine learning can support stress detection and the data set described here could advantageously be analyzed by these methods in the future. Additionally, the application of SC signal deconvolution could allow us to separate tonic and phasic components of the SC signal, identifying phasic drivers underlying responses to in-game events [36]. This could allow us to develop personalized, event-based PTSD profiles that integrate information from the simulation context into the stress detection process. Finally, more advanced methods of multimodal signal fusion could enable a better characterization of the stress responses through the combination of the SC and BVP/HR signals, possibly yielding a more satisfactory cross-modality combination and a more accurate model of the patients' stress responses [37].

\section{Conclusion}

In this study we used StartleMart, a game-based PTSD exposure therapy and stress inoculation therapy tool, to elicit stress responses from 14 male PTSD patients. We collected physiological indications of stress responses from skin conductance and blood volume pulse, along with external PTSD profile information indicating PTSD symptom severity as well as self-reports of experienced stress as sources of ground truth. From the physiological signals, 45 individual features were extracted and correlated to the sources of ground truth. The results of the analyses in this paper indicate that physiological responses to StartleMart are highly correlated with PTSD symptom severity and subjective experience expressed 
through self-reports of stress. Additionally, an application of principal component analysis to reduce the number of features into two distinct components suggests that two response patterns are manifested in relation to the content presented in the simulation: One which is primarily related to stress resilience/para-sympathetic activity and exhibits a negative correlation to external measures of PTSD symptom severity and one which is primarily related to stress sensitivity/sympathetic activity and exhibits a positive correlation to external measures of PTSD symptom severity. This underlines the complex nature of user responses to rich stimulus presenting simulations and motivates the further use and study of multiple modalities for capturing stress responses. Further, the fact that StartleMart elicits stress responses with PTSD patients lends credence to the general idea of using game-based stimuli of every-day life situations for stress inoculation training for PTSD patients. However, any treatment efficacy is unknown at this point and would require a randomized study. Nonetheless, the fact that physiological responses seem to scale with measures of symptom severity, self-reports and an indicator of recovery over time, indicates a promise to using stress eliciting game-based solutions like StartleMart for diagnosis and treatment of PTSD. Future work will focus on leveraging these findings to refine profiling and adaptive game-based solutions supporting diagnosis and treatment in psychiatric work.

Acknowledgements This research was supported by the Danish Council for Technology and Innovation under the Games for Health project and by the FP7 ICT project ILearnRW (project no: 318803).

\section{References}

1. C.W. Hoge, et al., New England Journal of Medicine 351(1), 13 (2004)

2. E.B. Foa, et al., Effective treatments for PTSD, practice guidelines from the International Society for Traumatic Stress Studies, 2nd edn. (New York: Guilford Press, 2009)

3. C. Holmgård, G.N. Yannakakis, K.I. Karstoft, H.S. Andersen, in ACII. IEEE (Springer, 2013), pp. 523-528

4. M. Siepmann, et al., Psychopharmacology 168(3), 293 (2003)

5. D.P. Wood, et al., Studies in health technology and informatics 163, 696 (2011)

6. R.A. Calvo, S. D'Mello, IEEE Transactions on Affective Computing 1(1), 18 (2010)

7. R.W. Picard, E. Vyzas, J. Healey, IEEE PAMI 23(10), $1175(2001)$

8. H.P. Martínez, M. Garbarino, G.N. Yannakakis, in ACII (Springer, 2011), pp. 267-276

9. S. Popović, et al. Stress inoculation training supported by physiology-driven adaptive virtual reality stimulation (2009)

10. J.A. Healey, R.W. Picard, IEEE ITS 6(2), 156 (2005)
11. J. Hernandez, R.R. Morris, R.W. Picard, in ACII (Springer, 2011), pp. 125-134

12. C. Perala, Galvanic skin response as a measure of soldier stress. Tech. rep., DTIC Document (2007)

13. J.L. Andreassi, Psychophysiology: Human behavior and physiological response (Routledge, 2000)

14. W. Boucsein, Electrodermal activity (Springer Verlag, 2011)

15. S. Tognetti, et al., in ACII (Springer, 2011), pp. 101-110

16. G.N. Yannakakis, J. Hallam, in ACII (Springer, 2011), pp. $437-446$

17. N. Pole, Psychological Bulletin 133(5), 725 (2007)

18. J. Blechert, et al., Psychosomatic Medicine 69(9), 935 (2007)

19. T.B. Kashdan, et al., Behav. therapy 41(3), 350 (2010)

20. A.H. Kemp, D.S. Quintana, M.A. Gray, K.L. Felmingham, K. Brown, J.M. Gatt, Biological Psychiatry 67(11), 1067 (2010)

21. M.B. First, et al., Structured Clinical Interview for DSMIV-TR Axis I Disorders, Research Version, Patient Edition. $(S C I D-I / P)$ (Biometrics Res., New York State Psychiatric Institute, New York, 2002)

22. E.B. Blanchard, J. Jones-Alexander, T.C. Buckley, C.A. Forneris, Behaviour Research and Therapy 34(8), 669 (1996)

23. J. Wolpe, The practice of behavior therapy (Pergamon Press New York, 1973)

24. G. Yannakakis, J. Hallam, International Journal of Human-Computer Studies 66(10), 741 (2008)

25. R. Picard, E. Vyzas, J. Healey, IEEE Transactions on Pattern Analysis and Machine Intelligence 23(10), 1175 (2001)

26. J. Goldberger, S. Challapalli, R. Tung, M. Parker, A. Kadish, Circulation 103(15), 1977 (2001)

27. G.N. Yannakakis, J. Hallam, International Journal of Human-Computer Studies 66(10), 741 (2008)

28. G. Moody, in Proceedings of Computers in Cardiology (IEEE, 1993), pp. 715-718

29. M. Malik et al., Circulation 93(5), 1043 (1996)

30. M. Kendall, Rank Correlation Methods, 4th edn. (Griffin, London, 1970)

31. M.D. Robinson, G.L. Clore, Psychological Bulletin 128(6), 934 (2002)

32. G.N. Yannakakis, J. Hallam, International Journal of Human-Computer Studies 66(10), 741 (2008)

33. K.M. Magruder, B.C. Frueh, R.G. Knapp, M.R. Johnson, J.A. Vaughan, T.C. Carson, D.A. Powell, R. Hebert, Journal of traumatic stress 17(4), 293 (2004)

34. U.R. Acharya, K.P. Joseph, N. Kannathal, C.M. Lim, J.S. Suri, Medical and Biological Engineering and Computing 44(12), 1031 (2006)

35. J. Zhai, et al., in SoutheastCon, 2005. Proceedings. IEEE (IEEE, 2005), pp. 415-420

36. M. Benedek, C. Kaernbach, Psychophysiology 47(4), 647 (2010)

37. H.P. Martinez, Y. Bengio, G.N. Yannakakis, Computational Intelligence Magazine, IEEE 8(2), 20 (2013) 